\title{
Le rapport Duclert et le filtre des lendemains génocidaires
}

\author{
Marie-Eve Desrosiers
}

Citer cet article : Desrosiers Marie-Eve (2021), "Le rapport Duclert et le filtre des lendemains génocidaires », Revue d'Histoire Contemporaine de l'Afrique, Dossier : Au-delà du rapport Duclert, 9-27, en ligne. URL : https://oap.unige.ch/journals/rhca/article/view/rwandadesrosiers

Mise en ligne : 08 novembre 2021

DOI : https://doi.org/10.51185/journals/rhca.2021.e577

\section{Résumé}

La commission de recherche sur les archives françaises relatives au Rwanda et au génocide des Tutsi, ou commission Duclert, s'est récemment penchée sur l'engagement de la France au Rwanda, en cherchant également à contribuer à la compréhension historique des processus menant au génocide perpétré contre les Tutsi au Rwanda en 1994. Le rapport qu'elle a produit propose toutefois une lecture problématique des événements au Rwanda au début de la décennie 1990. Cet article soutient que, faisant du génocide la clé de lecture du Rwanda, ce rapport filtre ce qui s'est passé au Rwanda à la seule lumière des violences génocidaires de 1994. Afin de démontrer ce «filtre génocide », le propos s'articule autour de deux illustrations tirées de l'interprétation que la commission Duclert fait du début de la guerre qui entame la période dite de radicalisation au Rwanda en octobre 1990. II s'agit de la tendance à culpabiliser les autorités rwandaises à rebours, ainsi que d'une lecture "exceptionnaliste » faite des motivations (quasi ataviques) et des relations entre les acteurs de l'époque (nécessairement hors du commun) dès 1990. L'article conclut sur l'importance de comprendre I'histoire du génocide en revenant au contexte, et ainsi à la nuance et l'ambiguïté, des événements de 1990.

Mots-clés : Rwanda ; génocide ; Duclert ; France 
La Commission de recherche sur les archives françaises relatives au Rwanda et au génocide des Tutsi qui s'est récemment penchée sur l'engagement de la France au Rwanda a dû opérer avec des limites importantes. Dans un contexte politisé et divisé, elle a dû produire son rapport en un an et demi, une échéance rendue encore plus complexe par la multiplication des fonds d'archives à explorer et par la pandémie mondiale de Covid-19. Ses objectifs étaient par ailleurs ambitieux: trancher sur le rôle de la France dans le cadre de l'avènement du génocide perpétré contre les Tutsi en $1994^{1}$ et, ainsi, contribuer à notre compréhension des événements ayant mené à cette tragédie. Ces objectifs devaient toutefois être réalisés en adoptant un regard bien limité. La commission ne devait couvrir que la période allant du début de la guerre entre les autorités rwandaises et le Front/Armée patriotique rwandais·e (FPR/APR) à partir du $1^{\text {er }}$ octobre 1990 au génocide débutant le 6/7 avril et s'achevant mi-juillet 1994 . Toutefois, la plus grande limite de l'entreprise historique que constituait la commission Duclert, du nom de son président, I'historien Vincent Duclert, était sa composition. Largement constituée d'historiens et de juristes, elle ne comportait pas de spécialistes du Rwanda, de I'Afrique, ou encore de la politique et des relations internationales ${ }^{2}$. La plupart de ses membres partageaient néanmoins un intérêt de recherche commun, celui d'étudier des événements extraordinaires, qu'il s'agisse de crises, de guerres ou de génocides.

Ces derniers points ne sont pas une critique originale de la commission Duclert. De nombreux chercheurs, travaillant entre autres sur le Rwanda, ont décrié ces choix et surtout la composition de la commission ${ }^{3}$. C'est une critique que je partage, puisque cette composition a nécessairement impacté le travail de la commission et donc ses conclusions. Le profil des membres de la commission s'est notamment traduit par un biais important : celui de faire du génocide la seule clé de compréhension du Rwanda.

Cela peut sembler contre-intuitif de critiquer le fait de partir du génocide et de chercher à en retracer les caractéristiques et la mécanique (rôle de l'État et ses agents au service d'un plan d'extermination, obsession de l'identité, etc.) dans les années l'ayant précédé afin d'éclairer ce qui s'est passé en 1994. Toutefois, comme je l'ai expliqué ailleurs et comme d'autres rwandanistes l'ont affirmé avant moi $^{4}$, ce point de départ comporte le risque de n'interpréter et filtrer ce qui a précédé qu'à la lumière de ce qui a suivi. II s'agit d'une lecture de l'histoire à rebours ou qui s'apparente, comme j'en ai déjà parlé sur la base des travaux de Richard Reid, à une forme de «présentisme » de la crise ou de l'urgence ${ }^{5}$. Cette critique a

\footnotetext{
1 II s'agit du nom, officialisé par différentes instances, du génocide se produisant au Rwanda en 1994. Sans nier la nature de ce génocide, et le fait que ses victimes étaient tutsi, des formules alternatives plus courtes sont utilisées dans le texte afin de faciliter la lecture.

2 Il est toutefois à noter que Sylvie Humbert a publié sur les tribunaux gacaca mis en place au Rwanda pour traiter des crimes liés au génocide à une échelle locale. La commission comptait également une spécialiste de l'histoire diplomatique sous le général de Gaulle, Chantal Morelle.

${ }^{3}$ C'est entre autres le cas d'André Guichaoua dans « Rwanda : des commémorations du génocide rythmées par les agendas politique et diplomatique ", The Conversation, 25 avril 2021, en ligne. URL : https://theconversation.com/rwanda-des-commemorations-dugenocide-rythmees-par-les-agendas-politique-et-diplomatique-159078 (consulté le 15 juin 2021).

${ }^{4}$ Desrosiers Marie-Eve (2014), "Rethinking Political Rhetoric and Authority During Rwanda's First and Second Republics 》, Africa, 84(2), pp. 199-225 ; Desrosiers Marie-Eve (2020), " "Making Do" with Soft Authoritarianism in Pre-Genocide Rwanda », Comparative Politics, 52(4), pp. 557-579; Newbury Catharine et Newbury David (2000), «Bringing the Peasants Back In: Agrarian Themes in the Construction and Corrosion of Statist Historiography in Rwanda », American Historical Review, 105(3), pp. 832-877.

${ }^{5}$ Desrosiers Marie-Eve et Russell Aidan (2020), « Histories of Authority in the African Great Lakes: Trajectories and Transactions », Africa, 90(5), p. 953. Pour les travaux de Richard Reid, il est possible de consulter : (2011), « Past and Presentism: the 'Precolonial' and the Foreshortening of African History », The Journal of African History, 52(2), pp. 135-155.
} 
d'ailleurs été soulevée par I'historien Frédéric Bozo lorsqu'il parlait du « regard rétrospectif » de la commission Duclert dans un article paru au moment d'écrire ce propos ${ }^{6}$. Même si l'histoire à rebours a l'avantage de raconter un parcours historique comme s'il était une ligne bien droite et simple, cette lecture est réductrice puisqu'elle n'aligne et ne considère que ce qui cadre avec cette ligne, ou ce regard établi rétrospectivement. La lecture proposée par le rapport Duclert n'est ainsi que l'une des différentes interprétations que l'on peut donner aux événements au Rwanda entre 1990-1994. Forte des limites de la commission, cette interprétation s'est toutefois largement réduite à lire l'ensemble des événements par le filtre de leur point d'arrivée fatidique : le génocide.

Afin d'illustrer mon propos, et les plis d'une lecture filtrée par le génocide, je me pencherai spécifiquement sur l'interprétation que le rapport fait des événements entourant I'attaque du Rwanda par le FPR/APR en octobre 1990, un épisode qui marque le début de la guerre entre le gouvernement rwandais et les attaquants. La première partie de mon texte explicite ma critique, en la situant plus largement dans les recherches sur les années précédant le génocide. Mon propos se tourne ensuite vers deux illustrations de l'argument : la tendance à culpabiliser les autorités rwandaises à rebours, ainsi que la lecture " exceptionnaliste » faite des motivations (quasi ataviques) et des relations entre les acteurs de l'époque (nécessairement hors du commun) dès 1990.

\section{L'argument : une lecture sur la base d'un « filtre génocide »}

Pourquoi se concentrer sur ces quelques mois de la fin de l'année 1990 plutôt que de jeter un regard plus large sur l'ensemble de la période couverte par le rapport ? Tout d'abord, ces quelques mois constituent un moment charnière ou une "jonction critique ", comme les appellent plus communément les politistes ${ }^{7}$ : ils lient l'avant-guerre et la guerre qui s'ensuit jusqu'au génocide. Pour ceux qui cherchent à retracer à travers l'histoire rwandaise la montée d'identités qui tuent, ces mois sont le moment où la " mécanique génocidaire " se met en branle et où les réalités rwandaises basculent en dehors de l'ordinaire. Cette idée est d'ailleurs implicitement évoquée par la notion de « radicalisation »souvent associée sans qu'on ne la questionne à cette période allant du début de la guerre au génocide ${ }^{8}$. Néanmoins, les études les plus fines des motivations dans le cadre du génocide ont largement contredit la thèse de l'endoctrinement et de la radicalisation, ou du moins son rôle comme motif unique derrière la perpétration des atrocités au Rwanda' . Si des versions radicales de l'identité ont effectivement

\footnotetext{
${ }^{6}$ Frédéric Bozo, « II faut considérer le rapport Duclert non comme l'aboutissement mais comme le point de départ d'un véritable travail historique », Le Monde, 16 mai 2021, en ligne. URL : https://www.lemonde.fr/idees/article/2021/05/16/il-faut-considerer-lerapport-duclert-non-comme-l-aboutissement-mais-comme-le-point-de-depart-d-un-veritable-travail-historique_6080347_3232.html (consulté le 15 juin 2021).

7 Sur ces «jonctions critiques », voir notamment Capoccia Giovanni (2015), "Critical Junctures and Institutional Change », in J. Mahoney et K. Thelen (dir.), Advances in Comparative-Historical Analysis, Cambridge, Cambridge University Press, pp. 147-179. L'idée des jonctions critiques est de se pencher avant tout sur les points saillants d'une tendance ou d'un phénomène, afin d'en tirer une compréhension causale plus évidente, tout en évitant les distractions que pourraient constituer des points ou faits jugés moins importants.

8 Pour une discussion des débats entourant la notion de « radicalisation » dans les contextes de violences, incluant le Rwanda, voir notamment McDoom Omar Shahabudin (2020), «Radicalization as Cause and Consequence of Violence in Genocides and Mass Killings », Violence: An International Journal, 1(1), pp. 123-143.

9 Voir par exemple Fujii Lee Ann (2009), Killing Neighbors. Webs of Violence in Rwanda, Ithaca/Londres, Cornell University Press ; Mironko Charles (2004), " Igitero: Means and Motive in the Rwandan Genocide », Journal of Genocide Research, 6(1), pp. 47-60. Sur
} 
circulé de manière significativement accrue à partir de 1990, nous ne pouvons affirmer une conversion totale des esprits rwandais à l'ethnisme ou encore que l'identité était la principale variable opérante des massacres lors du génocide.

Ce moment est donc primordial dans notre compréhension du génocide, tout en étant un moment à la source de débats importants chez les chercheurs. C'est un moment qui finit en effet par opposer ceux qui y voient le point de rupture et de basculement dans l'identitaire meurtrier, et ceux qui insistent au contraire sur l'importance de le replacer dans un cadre, notamment politique, plus large pour comprendre les forces et motivations complexes en jeu. C'est une période qui oppose certaines interprétations produites rapidement dans la foulée du génocide et insistant sur les médias radicaux ou "l'identification de l'ennemi », et donc sur l'idéologie comme véhicule d'un crime identitaire, aux lectures venues par la suite sur la base de recherches empiriques approfondies, auprès des tueurs notamment. Ces dernières lectures insistent sur des éléments plus divers pour expliquer le génocide : la peur née de la guerre, les divisions au sein de la classe politique rwandaise, la remise en question de l'ancien appareil de gouvernement, la défense des acquis pour certaines franges politiques, etc.

L'identité a bien entendu joué un rôle dans les événements au Rwanda au début des années 1990, mais jamais de manière simple et totale comme certaines interprétations instrumentalistes ont pu le suggérer, avec des élites agitant la haine de l'autre et des populations les suivant sans poser de questions ${ }^{10}$. Les motifs pour soutenir la violence ou même pour la produire ont été, à tout moment, complexes. Le contexte rwandais est plus riche que la seule lecture identitaire ne le suggère. La relation à l'identité y a toujours été variable, même si parfois hautement politisée, sans jamais signifier une adhésion complète à un ethnocentrisme radical de la part de tous les Rwandais, y compris de la classe politique. La question identitaire est « piégée » au Rwanda, comme le rappelait Claudine Vidal, et l'analyste étranger a régulièrement été pris à ce piège, voyant en l'identité une clé d'interprétation pleine de sens à la lumière des manifestations de la violence, alors que cette grille d'interprétation est trop réductrice ${ }^{11}$.

C'est donc ce moment charnière, complexe et contesté, qui constitue le point d'entrée du rapport Duclert, d'où son importance pour saisir les bases de l'entreprise historique de la commission. Cette dernière connaît-elle les débats entourant la période ? II est difficile d'en juger. Le rapport soulève néanmoins dès le début certaines lacunes quant à la capacité des chercheurs de la commission à s'appuyer sur les travaux existants ${ }^{12}$. Qu'elle ait connu ou non

\footnotetext{
cette causalité complexe, voir aussi McDoom Omar Shahabudin (2021), The Path to Genocide in Rwanda. Security, Opportunity, and Authority in an Ethnocratic State, Cambridge, Cambridge University Press.

${ }^{10}$ C'est notamment l'explication que l'on retrouve implicitement ou explicitement dans les travaux portant sur le rôle de l'identité ou des médias, par exemple Uvin Peter (1997), "Prejudice, Crisis, and Genocide in Rwanda », African Studies Review, 40(2), pp. 91-115 ou encore Chrétien Jean-Pierre (1995), "Rwanda : la propagande du génocide », in Reporters sans frontières (dir.), Les Médias de la haine, Paris, La Découverte, pp. 22-56.

11 Vidal Claudine ([1985] 2005), "Situations ethniques au Rwanda », in J.-L. Amselle et É. M'Bokolo (dir.), Au coeur de l'ethnie. Ethnies, tribalisme et État en Afrique, Paris, La Découverte, pp. 167-184. Voir aussi Lemarchand René (2009), The Dynamics of Violence in Central Africa, Philadelphia, University of Pennsylvania Press.

12 Les membres de la commission sont conscients de la politisation des débats entourant le Rwanda, mais semblent n'avoir pas pu largement consulter les travaux existant sur la période. Commission de recherche sur les archives françaises relatives au Rwanda et au génocide des Tutsi (2021), La France, le Rwanda et le génocide des Tutsi (1990-1994), Paris, Armand Colin, p. 17. Ci-après Rapport Duclert.
} 
ces débats, on sent dès ce point d'entrée les plis du rapport Duclert, notamment son appartenance au « camp » de la lecture plus simpliste et linéaire du processus vers le génocide.

Le premier chapitre ambitionne en effet de tracer une ligne droite entre les premiers jours couverts, ceux qui suivent l'attaque du FPR/APR en octobre 1990, et le génocide. Le rapport fait implicitement sien le narratif du passage à l'identité qui tue, proposant une interprétation à rebours du génocide largement tissée autour d'autorités obsédées par l'identité - une interprétation qui fait écho par ailleurs à l'interprétation historique promue par les actuelles autorités rwandaises. Cette lecture s'articule également autour d'un basculement dans le fatidique et l'exceptionnel, malgré un clin d'œil plus nuancé au contexte politique rwandais dans les dernières pages du chapitre.

Or, malgré les signaux quant aux risques de violence ethnique importants ${ }^{13}$, personne ne savait en 1990 qu'un génocide se produirait au Rwanda. L'obsession des autorités rwandaises pour l'identité et la mécanique génocidaire ne peuvent simplement être prises pour acquises et retrouvées dès ce moment de l'histoire rwandaise. Autrement dit, il faut comprendre ce qui crée les génocidaires plutôt que les imaginer uniquement sous l'angle de ce qu'ils deviendront. De même, si de manière rétrospective on s'imagine, avant l'heure, tous les acteurs au Rwanda comme pris dans une marche vers le génocide, on finit par voir tout geste, tout choix, comme faisant nécessairement partie de cette catastrophe exceptionnelle. II faut plutôt jeter un regard dans le sens de l'histoire: comprendre le contexte et surtout le devenir génocidaire plutôt qu'en faire un point de départ est essentiel à la compréhension de ce qui s'est passé au Rwanda entre 1990 et 1994. Le reste du propos s'attarde à illustrer ce « filtre génocide » qu'a fait sien la commission Duclert.

\section{Infamie et culpabilité à rebours}

Le FPR/APR, composé principalement de quelques milliers d'exilés et réfugiés rwandais en Ouganda, attaqua le Rwanda par sa frontière nord le $1^{\text {er }}$ octobre 1990. L'attaque fut suivie d'une riposte militaire de la part des Forces armées rwandaises (FAR), appuyées par quelques alliés, dont la France. L'attaque fut également l'occasion d'une vraisemblable mise en scène par le gouvernement rwandais d'échanges de tirs ou d'un risque accru d'une action militaire du FPR/APR à Kigali. Ces échanges servirent de prétexte à des rafles à travers le pays dans les jours qui suivirent, dont de nombreux Tutsi firent les frais ${ }^{14}$. Ces événements marquèrent le début d'un conflit armé intermittent qui opposa le FPR/APR aux FAR, et qui se termina par la victoire du FPR, marquant également la fin du génocide en juillet 1994. Dans le retour que propose le rapport Duclert sur les événements des derniers mois de l'année 1990, on sent toutefois un préjugé défavorable envers les autorités de l'époque à Kigali, souvent incarnées par le président Habyarimana.

\footnotetext{
13 Plusieurs observateurs ont noté à l'époque le risque d'un ciblage violent des Tutsi dans la foulée de l'attaque du FPR/APR. Le rapport Duclert mentionne d'ailleurs un document de l'attaché de défense français datant de novembre 1990 dans lequel une annotation manuscrite soulève le risque de pertes de vie à la hauteur de 500000 à 700000 Tutsi si le gouvernement rwandais ne récupère pas les territoires perdus face au FPR/APR. Rapport Duclert, p. 76.

${ }^{14}$ Sur ces événements, on peut consulter entre autres Guichaoua André (2010), Rwanda, de la guerre au génocide. Les politiques criminelles au Rwanda (1990-1994), Paris, La Découverte.
} 
D'entrée de jeu, il est important de noter que le gouvernement en place à l'époque au Rwanda n'est pas le même que celui à la tête du pays au moment où le génocide commence en avril 1994, ce qui rend l'assimilation des autorités de l'époque à celles en place lors du génocide plus que problématique. Le président Habyarimana est mort avant le début du génocide et les membres du gouvernement intérimaire qui se met en place dans la foulée de sa mort ne sont pas ceux du gouvernement en 1990. Une série de gouvernements sont d'ailleurs portés au pouvoir entre 1990 et le début du génocide. Cette succession rapide de gouvernements au cours de ces quatre ans est notamment le fait d'un processus de libéralisation de la sphère politique rwandaise à partir de 1991.

Il est par ailleurs important de noter que, bien que de manière rétrospective on ait parfois tendance à envisager le cadre politique rwandais comme assez homogène, que ce soit parce qu'on lui attribue une tendance " ethnicisante » pro-hutu depuis l'indépendance ou un visage autoritaire hostile à toute dissension, les acteurs politiques rwandais n'ont en fait jamais constitué un bloc uni. Les dissensions existaient au sein même des gouvernements quant à la ligne politique à mener, y compris sur la question des identités, même si ces gouvernements étaient clairement à forte majorité hutu. Ces divisions ont été à la source de crises internes ponctuelles, alimentées parfois par une tension entre modérés et radicaux de la cause identitaire, mais également par d'autres clivages importants comme le régionalisme ou les prédilections politiques ${ }^{15}$.

\section{Définir les méchants et les bons}

Cette contextualisation est importante parce qu'elle tranche avec la lecture qui est celle du rapport Duclert. Les autorités rwandaises telles qu'elles sont présentées dès les premiers moments des événements de 1990 y sont traitées de manière globalisante, unies derrière un pli identitaire commun. En d'autres termes, les autorités rwandaises semblent porter dès octobre 1990 la culpabilité de ce que leurs successeurs décideront à l'aube du génocide. Bien entendu, ces autorités portent le poids des décisions qu'elles ont prises dans les jours qui ont suivi l'attaque, telles que l'organisation de rafles, incluant notamment des Tutsi dont des milliers sont rassemblés dans des conditions dramatiques, ou leur encouragement au ciblage des Tutsi par les populations rurales, nommé avec un certain euphémisme " auto-défense ». La commission Duclert ne fournit pas de chiffres concrets sur cette violence qui se dessine dans la foulée de l'attaque du FPR/APR. Elle la qualifie néanmoins de "violences extrêmes », un vocable qui donne matière à discussion comparativement à ce qui suivra en 1994. Le vocable " extrême » pour qualifier le ciblage des Tutsi en octobre 1990 ne suggère-t-il pas ce que le rapport conçoit être de manière téléologique un passage à la mécanique génocidaire?

C'est probablement la même perspective qui explique le traitement réservé aux autorités rwandaises de l'époque, " coupables » avant l'heure d'enclencher la marche vers le génocide. Le choix des mots pour qualifier les autorités rwandaises, dont le rapport soulève régulièrement le peu de crédibilité et la volonté manifeste de manipuler leurs alliés et bailleurs est signifiant.

\footnotetext{
15 Voir, notamment, Munyarugerero François-Xavier (2003), Réseaux, pouvoirs, oppositions : la compétition politique au Rwanda, Paris, L'Harmattan.
} 
À titre d'exemple, une section du rapport s'intitule «Mentir avec aplomb ${ }^{16}$ ». Des mots et expressions comme "induire en erreur», "convaincre » comme s'il s'agissait de flouer, " chantage », " mensonges", " esquiver», etc. apparaissent régulièrement dans le propos ${ }^{17}$. Ces qualificatifs pourraient en soi être ignorés ou considérés comme un choix stylistique pour animer le propos du rapport. Toutefois, ce genre de qualificatif est réservé aux seules autorités rwandaises et aux Français les appuyant. Ces derniers sont, de leur côté, décrits comme peu informés, naïfs ou « choisissant de croire » ce que Kigali leur dit, un vocabulaire qui contraste avec celui utilisé pour décrire la position de certains acteurs plus critiques des Rwandais au sein de l'appareil politique en France ${ }^{18}$.

Plus important encore que le choix des mots, il faut d'ailleurs noter le traitement réservé aux positions et interprétations proposées par les autorités rwandaises de l'époque, qui sont systématiquement rejetées par le rapport Duclert et perçues comme une forme de manipulation. Le rapport ne propose ainsi aucune exploration approfondie des bases de la position rwandaise (par-delà les causes possibles de manipulation : la nécessité d'obtenir des armes, de cacher leur implication dans les rafles anti-Tutsi, etc.), ni ne fait la démonstration de la justification de les rejeter. En d'autres termes, les arguments du gouvernement rwandais ne sont jamais explorés, ne serait-ce que pour faire la démonstration de leur manque de validité.

Le contraste avec le traitement réservé au FPR/APR est assez marquant. Ici, contrairement aux termes employés pour parler des autorités rwandaises, la commission avertit clairement ses lecteurs de l'importance du choix des mots. En mettant entre guillemets les mots " assaillants », "rebelles », " agression », " offensive » etc., le rapport semble vouloir laisser planer un doute quant à la nature de l'action du FPR/APR en octobre 1990. Ailleurs, le rapport semble lire avec scepticisme ces qualificatifs et leur implication: "Qualifiés d'agresseurs, de rebelles, puis de guérilleros ou de partisans, ils représentent un germe de déstabilisation dans un pays ami ${ }^{19}$ ». Or, de manière planifiée et organisée, le FPR/APR a bien mené une incursion armée sur le territoire rwandais. Il est à se demander quels termes la commission aurait préférés à ceux qu'elle met parfois entre guillemets pour décrire un groupe qui choisit de pénétrer sur un territoire de manière armée. D'une manière générale, on sent une retenue dans la caractérisation du FPR/APR, retenue qui tranche avec le traitement réservé aux autorités rwandaises. Le rapport tend ainsi à attribuer au FPR/APR des objectifs nobles, tels que la remise en question de l'autoritarisme au Rwanda (en citant d'ailleurs, il faut le mentionner, certains acteurs de l'époque), et à minimiser la force armée que ce même FPR/APR a déployée.

Comme pour les autorités rwandaises, cette caractérisation du FPR/APR se base toutefois davantage sur des affirmations que sur une réelle validation ou invalidation des positions. La commission tend largement à présenter la position et les aspirations politiques du FPR/APR telles que promues par le FPR/APR lui-même et reprises par certains acteurs à l'époque. Elle tend par ailleurs à rejeter les arguments sous-tendant la force et les bases du FPR/APR. C'est le cas du soutien possible au mouvement par l'Ouganda, pays d'où l'attaque provient et où bon nombre de membres du FPR/APR se sont exilés et/ou ont grandi et fait leurs classes

\footnotetext{
${ }^{16}$ Rapport Duclert, p. 97.

17 Par exemple, Rapport Duclert, pp. 36, 46, 47, 49, 63, 93, 96, 97, 98, 101, 113.

18 Rapport Duclert, p. 97.

19 Rapport Duclert, p. 51.
} 
militaires. L'hypothèse d'un appui ougandais est rejetée prestement, essentiellement sur la base d'une note de la DGSE (Direction générale de la Sécurité extérieure) publiée environ un mois après l'attaque ${ }^{20}$. La question n'est pas davantage explorée, y compris la possibilité d'un soutien tacite de la part du gouvernement ougandais, alors que l'histoire de la région suggère au contraire la régularité de ce genre d'implications de la part des pays voisins ${ }^{21}$.

\section{Concevoir la menace que fait peser l'attaque du FPR/APR}

Ce sont toutefois les discussions entourant la menace posée par l'attaque du FPR/APR qui traduisent plus particulièrement le biais implicite du rapport. Toute une série d'éléments entourant l'attaque du FPR/APR et surtout son importance en tant que menace militaire sont rejetés sans qu'ils ne soient réellement invalidés. La tendance générale est ainsi à minimiser l'attaque, qu'il s'agisse d'évoquer l'organisation plus large du FPR/APR ou la possibilité que celui-ci ait pu pénétrer en territoire rwandais avant l'attaque pour y développer des cellules de soutien et y cacher des armes - possibilité présentée par la commission comme une invention des autorités rwandaises pour exagérer la menace. Or les Français ne sont pas les seuls à noter, y compris dans les mois voire les années précédant l'attaque, la possibilité de certains mouvements de la part d'opposants, y compris sur le territoire rwandais. Si la question des caches d'armes est moins évidente à trancher - quoique les Belges au Rwanda à l'époque semblent avoir également considéré cette possibilité - plusieurs ex-affiliés du FPR et d'autres commentateurs ont parlé de contacts réguliers entre le FPR/APR et les populations rwandaises au pays : organisation de collectes de fonds en soutien à l'organisation, réseautage, etc. ${ }^{22}$. Les archives belges évoquent également la présence d'Alexis Kanyarengwe, opposant au gouvernement passé au FPR/APR, sur le territoire rwandais à la fin des années $1980^{23}$.

De manière similaire, lorsque certains acteurs français parlent de la propagande des groupes diasporiques liés au FPR/APR et de leur degré d'organisation, le ton du rapport Duclert devient dur. Ceux qui voient en la diaspora entourant le FPR/APR un groupe organisé sont discrédités. L'idée que les groupes diasporiques liés au FPR/APR feraient la promotion d'une interprétation spécifique des événements ou de la propagande est également rejetée de manière expéditive, sans qu'aucune justification ne soit proposée. Alors que les discours des autorités rwandaises sont nécessairement tenus pour politiques, pourquoi ne pas s'imaginer que c'est également le cas de leur opposants ? Quoique beaucoup reste à faire sur l'histoire du FPR/APR, il n'en demeure pas moins que ceux qui l'ont exploré ont trouvé une capacité d'organisation et de communication importante, même en étant exilés/réfugiés ${ }^{24}$.

\footnotetext{
20 Rapport Duclert, pp. 110-111.

${ }^{21}$ On peut penser, par exemple, aux années 1960, alors que le Burundi tolérait, parfois même activement, l'organisation de raids vers le Rwanda. Puisque avoir «fermé les yeux » est ce qui est reproché à la France par la commission Duclert, il est d'ailleurs assez ironique que ce genre de positionnement de la part de l'Ouganda n'ait pas été envisagé.

22 Concernant les caches d'armes sur le territoire rwandais, voir Archives diplomatiques belges (ADB), 18888 । 2, telex 676 de I'ambassade de Kigali, "Situatie-Rebellen», 4 octobre 1990. Pour les contacts, voir Kajeguhakwa Valens (2001), Rwanda: de la terre de la paix à la terre de sang. Et après?, Paris, Éditions Remi Perrin ; Prunier Gérard (1993), "Éléments pour une histoire du Front patriote rwandais », Politique africaine, 51, pp. 121-138. Aussi sur la base d'échanges avec des anciens du FPR/APR.

${ }^{23}$ Entre autres, ADB, 18888 I 2, telex 334 de l'ambassade de Kigali, « docteur Alois Sebiziga », 29 mai 1989.

${ }^{24}$ Voir entre autres Kuperman Alan J. (2004), "Provoking Genocide: A Revised History of the Rwandan Patriotic Front 》, Journal of Genocide Research, 6(1), pp. 61-84 ; Prunier G., « Éléments pour une histoire... », art. cité.
} 
Le rapport démonte aussi la notion même de menace. Elle est largement décrite comme le fait de l'exagération des autorités rwandaises. Selon le rapport Duclert, dès les premiers jours, ces autorités auraient gonflé le nombre de combattants ennemis et laissé faussement planer la possibilité de liens avec l'Ouganda, afin d'obtenir un appui dans leurs opérations militaires contre le FPR/APR. La toute première phrase du chapitre donne le ton, en qualifiant l'interprétation que font les autorités rwandaises de l'attaque d'«alarmiste ${ }^{25} »$. Afin de démontrer le manque de crédibilité de l'interprétation de la menace proposée par le gouvernement rwandais, le rapport cherche à estimer la taille de la force d'incursion, pour en conclure à une exagération de la part des autorités rwandaises. En somme, l'attaque était de moins grande ampleur que ce qui a été annoncé dans ses premiers jours ; aussi il est suggéré que le gouvernement a menti de manière stratégique, malgré le flou entourant les événements dans les premiers jours de la crise.

Il est toutefois intéressant de noter que, si le rapport Duclert s'efforce de minimiser l'ampleur de cette attaque, plusieurs documents qu'il cite tendent au contraire à la dépeindre comme un événement militaire important. Certains documents mentionnent ainsi la perte de territoire de la part des troupes rwandaises suite à l'attaque. D'autres évoquent la « fragilité de la situation militaire » ou soulignent un rapport de force "préoccupant » et même la perte d'un hélicoptère. Un autre encore indique que "l'offensive d'octobre a mis en lumière les lacunes de la petite armée rwandaise ${ }^{26} »$. Le 20 octobre 1990, les troupes zaïroises présentes sur le territoire rwandais en appui au gouvernement semblent avoir perdu une cinquantaine de soldats, ce qui précipite la décision zaïroise de se retirer ${ }^{27}$. Dès lors, s'il est peut-être exagéré de croire en une menace existentielle pour le territoire rwandais et ses autorités, l'attaque et ses suites ont eu des impacts militaires évidents. Pourquoi alors présenter l'attaque comme si peu importante, surtout dans les premières sections du chapitre?

Pour comprendre l'estimation que les autorités rwandaises ont pu faire de cette menace, il importe par ailleurs de la replacer dans un contexte plus large. Cette menace militaire s'est profilée dans un contexte de déstabilisation préexistante, ce qui a pu contribuer à la crainte du gouvernement de voir les bases de son contrôle lui échapper. Si le rapport Duclert explore ce contexte de déstabilisation politique au Rwanda précédant l'attaque d'octobre 1990, il n'en fait toutefois pas un élément explicatif des stratégies du gouvernement rwandais. Or, avant l'attaque, ce gouvernement était déjà dans une situation fragilisée. Des formes de contestation populaire de plus en plus évidentes se manifestaient contre le régime. À ce sujet, le rapport Duclert cite un observateur jugeant le régime dans une situation "d'essoufflement », avant même l'attaque d'octobre $1990^{28}$. Dans un contexte de crise économique, alimentée par des facteurs internationaux et la gestion du gouvernement rwandais, la famine était réapparue. À la fin des années 1980, la situation était dramatique au point d'encourager les populations à chercher refuge ailleurs. Les crises de migration et de gestion de la frontière s'étaient multipliées durant les années 1980 et les passages entre les pays s'étaient accélérés dans les

\footnotetext{
25 Rapport Duclert, p. 36.

26 Par exemple, Rapport Duclert, pp. 99, 118.

27 Rapport Duclert, p. 54.

28 Rapport Duclert, pp. 89, 103.
} 
mois précédant l'attaque, alors que des milliers de Rwandais quittaient le pays ${ }^{29}$. Le gouvernement rwandais était d'ailleurs en passe de conclure des accords sur la question au moment de l'attaque du FPR, contrairement à la version présentée par le rapport ${ }^{30}$. Dans ce contexte, l'évaluation subjective faite par le gouvernement rwandais de la situation est donc probablement à prendre en compte dans son évaluation de la menace tout autant que le nombre de troupes pénétrant le territoire rwandais. Pour un régime à la légitimité déjà vacillante, l'attaque armée par le FPR/APR a certainement constitué un moment politique dramatique.

En somme, on voit poindre dans le rapport cette tendance à vouloir convaincre de la culpabilité d'un probable coupable avant l'heure et à en faire la preuve en la retraçant dans tous ses gestes, et cela même si au temps zéro, en octobre 1990, nous n'étions pas nécessairement face aux mêmes acteurs qu'en 1994. La commission tend ainsi à chercher les génocidaires chez les autorités rwandaises dès 1990, plutôt que de comprendre le contexte de leur émergence et ce qui a influencé le devenir de ceux qui, à terme, deviendront génocidaires en 1994.

\section{L'exceptionnalisme à rebours}

Utiliser le génocide comme filtre d'analyse a un autre impact majeur : celui de chercher à faire ressortir des années précédant la violence, incluant ces quelques mois entourant l'attaque du $1^{\text {er }}$ octobre 1990, ce qui aurait pu faire naître l'événement exceptionnel qu'est le génocide. Avec le regard de ceux qui savent ce qui a suivi, le rapport s'attarde ainsi à illustrer l'exceptionnalisme de la politique et des relations qui se dessinent en amont du génocide, lequel exceptionnalisme n'est d'ailleurs presque jamais démontré. À trop le chercher pour expliquer une violence d'une ampleur inimaginable, on se demande si la commission n'en vient pas à imaginer cet exceptionnalisme là où d'autres verraient des processus plutôt ordinaires, même si ces derniers ont fini par générer des violences extrêmes.

\section{Des motivations exceptionnellement identitaires}

Cette lecture exceptionnaliste se retrouve d'abord dans la culpabilisation à rebours des autorités rwandaises dès 1990, présentées implicitement comme dominées par des motifs ethnicisants plutôt que par une quête de résilience politique. II en ressort soit l'image d'une culture politique exceptionnellement obsédée par l'identitaire au Rwanda, soit la description de l'attaque de 1990 comme une rupture exceptionnelle faisant basculer en l'espace de quelques mois le Rwanda dans une altérité radicale. Ni l'une ni l'autre de ces interprétations ne permettent de saisir la complexité des processus qui se mettent en branle dans les années 1990. Mais elles traduisent toutes deux une lecture de l'histoire à l'envers: sur la base d'un crime de masse exceptionnel, on cherche dans ce qui l'a précédé l'exceptionnellement

\footnotetext{
29 Guichaoua André, "Le problème des réfugiés rwandais et des populations banyarwanda dans la région des Grands Lacs africains 》, Genève, UNHCR, mai 1992. Toute une série de mouvements se dessinent entre les frontières rwandaises, ougandaises et tanzaniennes à l'époque, amenant des milliers de personnes à franchir les frontières.

30 Voir, entre autres, Guichaoua A., "Le problème des réfugiés... 》, op. cit.; République rwandaise, Présidence de la République, Commission spéciale sur les problèmes des émigrés rwandais, Le Rwanda et le problème de ses réfugiés. Contexte historique, analyse et voies de solution, Kigali, mai 1990.
} 
identitaire, un travers que l'on retrouve chez de nombreux chercheurs qui ont découvert le Rwanda à travers le génocide, et auquel n'échappe pas la commission. Catharine et David Newbury nous avertissaient déjà, il y a plus de 20 ans, quant à ce risque :

Leur introduction à la société et à ses habitants s'est faite par le seul biais du génocide, et leur expérience intellectuelle s'est souvent fortement inspirée des modèles de génocides précédents. Ainsi, les thèmes sur lesquels ils ont choisi de se concentrer - l'ethnicité, la violence et l'État étaient ceux qui étaient les plus évidents pour le génocide lui-même. ${ }^{31}$

Un des exemples les plus évidents est le passage sur le terme inyenzy (sic) dans le rapport $^{32}$. Généralement traduit en français par "cancrelat», ce terme est décrit par la commission comme une injure envers les Tutsi. Bien que ce commentaire apparaisse dans le cadre de la discussion des événements de 1990, la commission souligne à travers ce mot le lien entre le génocide et la déshumanisation de l'autre: "On injurie l'ennemi pour l'impressionner et pour se donner le courage de le poursuivre, le blesser, le tuer. Mais I'assimilation à un animal procède des mécanismes du génocide en ce qu'elle dénie à l'autre le statut d'être humain, autorisant toutes les transgressions ${ }^{33}$. "La réflexion que propose la commission autour de ce mot s'appuie d'ailleurs sur les théories du génocide et de la Shoah. Sans nier que le mot inyenzi et bien d'autres sont devenus des mots déshumanisants dans les années qui ont suivi, il aurait été important de contextualiser le terme dans le contexte rwandais, y compris dans le contexte de l'attaque d'octobre 1990, avant de faire ce saut intellectuel expéditif dans l'univers génocidaire.

Le mot inyenzia en effet un historique qu'il importe de soulever. Le terme émerge dans le cadre d'attaques récurrentes contre le territoire rwandais dans les années 1960. Ces attaques sont menées par des exilés et des réfugiés ayant quitté le pays dans la foulée des violences entourant les transformations politiques au Rwanda avant et au cours de l'indépendance. Inyenzi désignait dans le cadre de ces attaques les attaquants. À l'époque, certains observateurs suggéraient que c'étaient les attaquants eux-mêmes qui avaient choisi de se prénommer inyenzi, d'autres que le terme leur avait été affublé ${ }^{34}$. Dans les deux cas, toutefois, le terme se voulait à l'origine vraisemblablement une référence aux tactiques employées : ces attaquants se mouvaient agilement et de manière cachée, souvent la nuit. Les attaques contre le territoire rwandais, de différentes ampleurs, ont continué tout au long de la décennie 1960, amenant les autorités rwandaises - et d'autres d'ailleurs - à parler de terrorisme inyenzi ${ }^{35}$. Le terme est demeuré et son usage s'est élargi, finissant par connoter la subversion de manière plus large. Les autorités rwandaises sous la Première République (1962-1973) eurent même tendance à parler d'inyenzisme pour se référer à tout effort de déstabilisation du gouvernement. S'il s'agit certainement d'un terme qui marque l'altérité et le rejet, il est également associé à l'action d'attaquer ou de déstabiliser le territoire. Les parallèles entourant les attaques dans les années 1960 et l'attaque de 1990 sont évidents. On peut se demander si cette connotation historique n'aurait pas mérité d'être soulevée, dans le cadre de la renaissance

\footnotetext{
31 Newbury C. et Newbury D., « Bringing the Peasants Back In... », art. cité, p. 832. Traduction de l'auteure.

32 Rapport Duclert, p. 113.

33 Ibid.

34 Sur les débats entourant l'origine du terme, voir notamment Riot Thomas, Bancel Nicolas et Rutayisire Paul (2017), « Un art guerrier aux frontières des Grands Lacs. Aux racines dansées du Front Patriotique Rwandais », Politique africaine, 147, pp. 109-134.

35 Sur cette notion plus large et I'inyenzisme, voir Desrosiers M.-E., « Rethinking Political Rhetoric... », art. cité.
} 
du vocable « inyenzi » en 1990, plutôt que d'y lire uniquement un saut vers la déshumanisation génocidaire.

\section{Des relations hors du commun}

La lecture des relations diplomatiques de l'époque - pour n'explorer que celles-là ${ }^{36}$ - tend à être également exceptionnalisante. Elle se retrouve dans la lecture que fait le rapport Duclert des relations entre la France et le Rwanda. Le premier chapitre pose tantôt implicitement, tantôt plus explicitement la question de savoir pourquoi la France a continué à soutenir le régime après l'attaque d'octobre 1990 et les violences qui s'en sont suivies ${ }^{37}$. La commission semble suggérer que face à l'autoritarisme du gouvernement rwandais, et tout particulièrement à la lumière des exactions commises ou tolérées par le régime lors de la crise commençant en octobre 1990, la présidence et l'appareil administratif français auraient dû se désolidariser de leur allié. On en retire l'impression que ce soutien continu était problématique, face à un partenaire aux pratiques politiques de plus en plus condamnables. En d'autres termes, il s'agissait d'une forme de soutien hors norme. Le rapport cite à ce sujet l'avis du secrétariat général de la Défense nationale (SGDN), très critique du gouvernement rwandais. Ce dernier évoque à l'inverse l'intérêt d'entretenir les relations avec le FPR/APR; le rapport suggère également plus loin que les États-Unis auraient encouragé la France à s'intéresser au FPR/APR ${ }^{38}$. Dans le contexte des relations préexistantes entre la France et le Rwanda, dans le cadre d'une crise sécuritaire sur le territoire rwandais, l'appui continu de la France est-il toutefois si surprenant, si exceptionnel ? Même si on peut rétrospectivement condamner ce soutien, le questionner en 1990 revient à le questionner bien avant l'heure.

Le rapport Duclert tend de prime abord à minimiser la coopération au développement et l'intérêt de la France au Rwanda antérieurement à la guerre, de manière à faire ressortir l'exceptionalisme de l'intérêt témoigné en 1990. Or, l'intérêt de la France envers le Rwanda débute bien avant 1990. Contrairement à ce que semble suggérer le rapport, dans le contexte de l'ensemble de l'aide bilatérale octroyée au Rwanda dans les décennies précédant le génocide (1973-1993) ${ }^{39}$, la France a toujours été l'un des trois principaux bailleurs du pays, à l'exception des années 1983 et 1984 (en cinquième position pour ces deux années) ${ }^{40}$. Si le soutien français au Rwanda n'atteint pas celui de la Belgique, l'ancienne puissance coloniale, et pâlit en comparaison avec l'aide de la France dans ses anciennes colonies, il était, lorsqu'on le compare aux autres bailleurs bilatéraux dans le cadre rwandais, relativement soutenu et substantiel. S'arrêter uniquement aux montants ou encore à l'aide militaire, comme le fait le rapport, tend par ailleurs à ignorer des éléments importants du soutien français au cours de la Deuxième République, témoignant d'une volonté française de nourrir ses relations avec le Rwanda. On peut penser à l'appui fourni au secteur touristique par l'entremise du financement, dans les années 1970, d'un village touristique (le village Urugwiro hébergeant aujourd'hui la

\footnotetext{
36 Une réflexion similaire pourrait être articulée autour des relations militaires de l'époque entre Français et Rwandais, par exemple.

37 Rapport Duclert, p. 71.

38 Rapport Duclert, pp. 71, 122.

39 L'année 1994 n'est pas un bon point comparatif, puisque le génocide et l'effort humanitaire à déployer en réponse à celui-ci ont fortement influencé les processus d'aide au Rwanda.

40 Voir les données de la Banque mondiale, en ligne. URL : https://data.worldbank.org/indicator/DT.ODA.ALLD.CD?locations=RW (consulté le 15 juin 2021).
} 
présidence), au soutien important quant au développement des infrastructures routières, ou même à l'offre d'un avion au président rwandais, en passe d'être finalisée en 1990. Dans le contexte des autres partenariats de la France en Afrique, ces apports peuvent avoir été négligeables, mais vus depuis le Rwanda, ils étaient considérables.

Par ailleurs, à part une comparaison avec la Belgique, problématique du fait même que les Belges ont maintenu après l'indépendance un intérêt tout particulier pour le Rwanda en raison de leur relation historique ${ }^{41}$, la lecture des relations diplomatiques et de coopération ne situe pas l'action de la France dans un univers comparatif plus large. Or, si cela avait été fait, il aurait été noté que, dans le contexte de la crise d'octobre 1990 et des exactions qui ont suivi, la France a agi comme de nombreux bailleurs et alliés l'ont fait et le font encore envers leurs partenaires autoritaires, au Rwanda comme ailleurs. L'étude approfondie des pratiques de différents bailleurs au Rwanda produite par Peter Uvin souligne cette tendance très évidente dans les années précédant le génocide, à continuer à opérer de manière habituelle, même dans la période dite de radicalisation ${ }^{42}$. L'aide publique au développement accordée au Rwanda entre la guerre et le génocide a d'ailleurs augmenté plutôt que chuté, dans un contexte de crise alimentée par la guerre. Cela traduit la tendance bien ordinaire des relations d'aide envers le Rwanda. Face à une crise sécuritaire aux impacts humanitaires importants (déplacements de population à l'intérieur du pays), la communauté internationale a répondu présente.

Il importe d'ailleurs de noter que depuis le milieu des années 1970, l'aide offerte au Rwanda n'a jamais été exceptionnelle. Elle a toujours été dans la norme pour les pays privilégiés, les "aid darlings", ou pour les pays ayant connu des conflits ou des crises majeures $^{43}$. En d'autres termes, l'aide et les processus d'octroi et de gestion de l'aide au Rwanda, lorsque on les étudie d'un point de vue comparatif, cadrent avec ceux de pays favorisés par les bailleurs, ce que le Rwanda est devenu à partir de la Deuxième République (1973-1990/1994) et ce qu'il est certainement demeuré en raison de la taille de l'effort humanitaire engendré par le génocide. Le soutien continu de la France s'inscrit donc dans ce contexte plus large de soutien continu de la part des bailleurs, avant, pendant et après le génocide. Et ce, malgré les réticences des États-Unis et de l'Allemagne, soulevées par le rapport dans la foulée de l'attaque d'octobre 1990.

Dès lors, si la commission semblait s'attendre à une prise de position forte et frontale de la part de la France face à l'autoritarisme rwandais en amont du génocide et à la coercition qui s'y rattachait, ces attentes concordent peu avec les pratiques diplomatiques de l'époque et celles plus contemporaines ${ }^{44}$. Si I'histoire nous dit que l'on tolérait les régimes autoritaires

\footnotetext{
41 En effet, en termes de choix de comparaison, pourquoi choisir de comparer la France avec le pays qui risquait, en raison de l'expérience coloniale, d'avoir la relation la plus « exceptionnelle» avec le Rwanda ? Cela semble un choix qui allait nécessairement présenter l'apport français comme "secondaire ». D'autres comparaisons auraient pu être envisagées, comme les États-Unis ou I'Allemagne, également des bailleurs réguliers du Rwanda post-indépendance (rappelons toutefois que le Rwanda a été colonisé par I'Allemagne avant la Belgique pendant une courte période).

42 Uvin Peter (1998), Aiding Violence: The Development Enterprise in Rwanda, West Hartford, Kumarian Press.

43 À ce sujet, voir Desrosiers Marie-Eve et Swedlund Haley J. (2019), "Rwanda's Post-Genocide Foreign Aid Relations: Revisiting Notions of Exceptionalism », African Affairs, 118(472), pp. 435-462.

44 On peut, de manière plus contemporaine, penser aux relations tissées entre la France sous la présidence d'Emmanuel Macron et le Rwanda. Les questions de gouvernance ne sont pas ou peu soulevées dans le cadre de ces relations. Pour une version plus élaborée de l'argument sur les relations entretenues avec l'autoritarisme, voir Marie-Eve Desrosiers, " Authoritarianism has changed, and so should our approach to it ", Democracy in Africa, 3 juin 2021, en ligne. URL : http://democracyinafrica.org/authoritarianism-haschanged-and-so-should-our-approach-to-it// (consulté le 15 juin 2021).
} 
s'articulant autour d'un parti unique avant la fin de la guerre froide pour des raisons géostratégiques - ce que le discours de La Baule du président Mitterrand souhaitait voir changer en 1990 -, la période post-guerre froide n'a pas nécessairement changé cette tendance. Les pressions à la libéralisation ont existé en 1990 et continuent d'exister. Mais malgré les années dédiées à la promotion de la démocratie, à la bonne gouvernance, etc. la gestion de la relation avec un partenaire autoritaire tend encore aujourd'hui à demeurer oblique ou indirecte et non frontale. La raison en est simple: I'entreprise diplomatique demeure avant tout dédiée à maintenir les canaux d'échange et de bonnes relations. L'absence de critique ouverte de l'autoritarisme n'est surprenante ni au Rwanda à l'époque ou aujourd'hui, ni ailleurs ${ }^{45}$.

La France, tout comme d'autres bailleurs, n'a pas complètement ignoré les exactions au Rwanda en octobre 1990. Toutefois, la plupart des partenaires du Rwanda ont choisi de les aborder de manière non frontale. Par exemple, l'importance de l'ouverture politique a été soulevée dans le cadre de l'offre de soutien militaire en réponse à l'attaque, une "sorte de marché » comme l'indique le rapport où les fournitures militaires sont, espère-t-on à l'époque, un levier à la transformation politique ${ }^{46}$. Les pratiques mises en avant par la France dans le cadre de la crise se dessinant au Rwanda, allant d'appels à la modération par les canaux de diplomatie, à ce marchandage, étaient et demeurent certains des outils les plus communs dans la gestion oblique des relations avec des partenaires autoritaires. Si l'on attribue aujourd'hui une responsabilité rétrospective à la France pour n'avoir pas répondu plus clairement à I'autoritarisme au Rwanda, dès 1990, l'approche de la part de la France était en réalité plutôt commune et c'est une responsabilité qui est partagée par d'autres. On peut d'ailleurs se demander si, à la lumière des exactions commises par le FPR/APR dans le cadre de la guerre et après, et si l'actuel régime au Rwanda finissait par perdre la faveur dont il bénéficie, on ne revisiterait pas cette question.

Dans son effort de faire ressortir la nature problématique des relations françaises avec le Rwanda en 1990, la commission Duclert les interprète par ailleurs en faisant abstraction du cadre des relations et normes internationales dans lesquelles les acteurs français opèrent. Considérant que les relations diplomatiques sont fondamentalement des relations d'État à État, il semble étonnant de ne pas prendre en compte la violation du territoire rwandais comme un des éléments ayant pesé le plus lourd dans les discussions et décisions de l'époque. Les " amis » du Rwanda se sont largement focalisés sur cette violation du territoire que le pays venait de subir, dans un système international bâti avant tout autour du respect de la souveraineté étatique - conférant une autorité sur un territoire. Aussi problématiques que puissent avoir été jugées les autorités rwandaises de l'époque, notamment pour leur rôle direct et soutien tacite dans les rafles et l'auto-défense, il n'en demeure pas moins qu'elles étaient les autorités légalement reconnues d'un pays dont la souveraineté clairement établie venait d'être remise en cause. Or, la commission semble, en ces mois de 1990, tenir pour acquis que l'instinct et la politique humanitaires auraient dû prévaloir sur une lecture privilégiant la souveraineté et I'inviolabilité du territoire d'un État.

\footnotetext{
45 Voir entre autres Desrosiers M.-E. et Swedlund H. J., « Rwanda's Post-Genocide... », art. cité ; Hagmann Tobias et Reyntjens Filip (dir.) (2016), Aid and Authoritarianism in Africa: Development without Democracy, Londres, Zed Books.

${ }^{46}$ Rapport Duclert, p. 62.
} 
S'il faut d'ailleurs donner un exemple de l'inversion implicite dans cette lecture humanitaire quant à la pratique internationale, on peut penser aux événements de 1963-1964 au Rwanda. La comparaison est particulièrement éclairante. Encore plus qu'en 1990, ces événements ont montré l'importance de soutenir un allié lors d'une remise en question de son autorité sur son territoire, même dans le cadre d'exactions de sa part ou de la part d'autorités locales. En décembre 1963, sous le même régime international - dans un contexte de guerre froide où l'impératif quant aux relations amicales était plus fort - le territoire rwandais a été attaqué par un groupe largement composé d'exilés et de réfugiés. Cette attaque, tout comme en 1990, a donné lieu à des représailles importantes contre la population tutsi, au point que certaines d'entre elles, dans la région de Gikongoro, ont même été qualifiées de génocide ${ }^{47}$. Quoique ces violences contre la population aient été la source de pressions de la part des alliés du Rwanda, appelant les autorités rwandaises à la modération, il n'en demeure pas moins que le Rwanda a été appuyé militairement et logistiquement dans sa lutte contre les incursions, y compris par les États-Unis. La défense de la norme humanitaire par-delà la souveraineté a toujours été un positionnement exceptionnel, ou du moins réservé aux États en marge de la communauté internationale ou considérés comme parias, ce que le Rwanda n'était pas, ni en 1990, ni en 1963-1964.

C'est également sous cet angle qu'il faut aborder la propension du rapport à jeter un œil critique, et implicitement exceptionnaliste, sur ce que la commission appelle le «courtcircuitage » des « canaux réguliers » de la part du président Habyarimana dans ses contacts avec les autorités françaises. Quelles auraient dû être les attentes de la commission quant aux relations entre les autorités françaises et les autorités rwandaises ? Dans la section dédiée aux processus décisionnels, on voit poindre derrière le choix de rester au Rwanda une critique quant à la tendance aux contacts directs, fréquents et mêmes personnels entre les autorités rwandaises et l'Élysée. Le rapport relève la personnalisation des relations entre les deux présidents de l'époque, Habyarimana et Mitterrand.

Le verbe "court-circuiter » revient à deux reprises en l'espace de quelques pages, lorsqu'est décrit un président rwandais qui "court-circuite tous les échelons et tous les intermédiaires entre lui et François Mitterrand », ou encore un président rwandais et des chefs d'état-major qui s'adressent à l'échelon le plus élevé de manière à " court-circuiter les intermédiaires et leurs réticences ${ }^{48}$ ». Ailleurs, on évoque des communications fréquentes, comme les appels passés directement à François Mitterrand. Or, le rapport ne contextualise jamais, entre autres de manière comparative, ces contacts. Devrions-nous nous attendre à ce que des chefs d'État passent nécessairement par les canaux ordinaires et des intermédiaires ? Dans un cadre d'urgence, où le territoire d'un allié a été violé par une attaque armée, devrionsnous nous attendre à ce que le protocole plus conventionnel et les échelons administratifs au travers duquel il se profile en dehors de crises soient respectés? Au contraire, l'urgence tend à favoriser une politisation, soit une saisie des processus par les acteurs politiques plutôt que par les acteurs bureaucratiques ${ }^{49}$, et une personnalisation des relations. Cela s'est entre autres

\footnotetext{
47 Voir notamment Desrosiers Marie-Eve, Elusive Control: Pre-Genocide Rwanda's Authoritarian Trajectory, manuscrit en cours d'évaluation.

48 Rapport Duclert, pp. 118, 120.

49 Desrosiers M.-E. et Swedlund H. J., « Rwanda's Post-Genocide... », art. cité.
} 
reproduit dans la plupart des relations bilatérales avec le Rwanda à la suite du génocide, alors que certains acteurs, souvent des politiciens, ont réussi à passer en dehors des canaux réguliers pour apporter leur soutien au Rwanda ${ }^{50}$. Autrement dit, le court-circuitage des canaux réguliers est courant dans un contexte de crise. L'urgence crée un impératif à l'action rapide, qui ouvre un espace à une gestion plus personnelle et directe.

D'un point de vue purement institutionnel, concevoir la proximité et la personnalisation des liens entre deux présidents comme problématique, crise ou non, ignore par ailleurs le rôle central et donc personnel des présidents dans la politique étrangère de leur pays dans la plupart des régimes présidentiels. Les canaux réguliers et intermédiaires sont subordonnés à l'autorité présidentielle en matière de politique étrangère, et non l'inverse. Qu'il s'agisse de la France ou du Rwanda, selon leur constitution de l'époque, le chef d'État était l'acteur prééminent en matière de politique étrangère ${ }^{51}$. Ainsi, même en dehors de moments de crise, les présidents français ou rwandais détenaient le pouvoir de ces contacts directs ${ }^{52}$. Le rapport Duclert finit d'ailleurs par conclure à la légalité et à la normalité de voir un président français trancher en matière de politique étrangère. Sur la décision du président Mitterrand d'envoyer des parachutistes au Rwanda par exemple, le rapport affirme: "Il n'y a là rien que de normal ${ }^{53} »$. Sur les décisions récurrentes prises par le président Mitterrand en faveur de maintenir des troupes françaises au Rwanda, on indique qu'elles sont «instruites dans les formes ${ }^{54} »$. Ces passages apparaissent néanmoins après une longue recension des formes de proximité entre les deux gouvernements, tendant à laisser planer le doute sur la nature de ces relations, trop proches, trop personnelles.

En somme, si l'on ne peut nier la nature exceptionnellement violente du génocide, on ne peut présumer qu'il naisse de l'exceptionnel. C'est néanmoins cette tendance à la lecture exceptionnaliste que l'on retrouve dans le rapport Duclert, qu'il s'agisse de comprendre ce qui a animé les acteurs de l'époque ou de la forme qu'ont pris les relations entre eux. L'exceptionnel est la clé de lecture au point d'ignorer toute alternative, y compris le fait que ces processus se sont très certainement également inscrits dans des pratiques et des cadres usuels, connus et largement utilisés.

Rien de l'interprétation alternative que je propose ne change le point d'arrivée des années étudiées par la commission Duclert, c'est-à-dire le massacre des Tutsi en 1994. Mon interprétation ne remet pas non plus fondamentalement en question la conclusion ultime du rapport Duclert : fermer les yeux sur l'autoritarisme ou du moins des pratiques autoritaires et/ou coercitives d'un partenaire finit par être une forme de cautionnement tacite du régime, de ses pratiques et peut-être même, éventuellement, des dérives meurtrières qu'elles permettent. La commission a raison de voir une responsabilité par omission. Cette omission

\footnotetext{
50 /bid.

51 On peut consulter les constitutions des deux pays à ce sujet.

52 Pour la France, voir entre autres Kessler Marie-Christine (1999), La Politique étrangère de la France. Acteurs et processus, Paris, Presses de Science Po, p. 25; Anderson Malcolm (1970), Government in France: An Introduction to Executive Power, Oxford/Toronto/New York, Pergamon Press.

53 Rapport Duclert, p. 124.

54 Rapport Duclert, p. 125.
} 
est toutefois interprétée comme naissant d'un cadre prédestiné au génocide, alors que la réalité est beaucoup plus complexe.

Mon propos cherche en somme à illustrer les impacts de lectures présentistes ou à rebours comme celle, implicite, du rapport Duclert. Après tout, la commission avait non seulement pour but de trancher sur le rôle de la France au Rwanda au cours de ces années mais également de « contribuer au renouvellement des analyses historiques sur les causes du génocide des Tutsi, profondes et plus conjoncturelles ${ }^{55} »$. Elle avait, en d'autres termes, pour objectif de produire un savoir historique. C'est à ce niveau que je prends à partie le rapport. En faisant du point d'arrivée fatidique de la période la clé de lecture ultime de l'histoire rwandaise, on en propose une interprétation nécessairement filtrée par ce point d'arrivée. Les faits, événements, relations et processus dont discute la commission pour la période entourant l'attaque de 1990 ne sont peut-être pas faux, mais ils ne sont lus qu'à l'aune du génocide. L'interprétation qui est faite des gestes et politiques des autorités rwandaises suggère qu'ils sont nécessairement génocidaires avant l'heure, que les gestes et politiques de tout Français au Rwanda ou travaillant sur le Rwanda, sont nécessairement problématiques avant l'heure à moins qu'ils ne se soient opposés aux autorités rwandaises.

L'assertion à rebours est problématique en soi. Mais elle est également la principale méthode qui a été utilisée pour soutenir l'argument : I'affirmation devient démonstration face aux alternatives. Lorsque des interprétations dissonantes existent, surtout de la part d'acteurs de l'époque, elles sont rejetées par la commission sans qu'on fasse preuve de leur manque de fondement. La commission défend également son interprétation sur la base d'affirmations qui auraient méritées d'être contextualisées et validées du point de vue d'une compréhension plus fine du contexte historique, ainsi que d'un angle comparatif. Ces deux failles étaient pour le moins prévisibles à la lumière des limites de la commission, allant de son mandat à sa composition.

Ma critique constitue ainsi un appel à une lecture plurielle, sociologique et politiste, tout autant qu'historienne des événements précédant le génocide. L'écriture de l'histoire ne peut s'accomplir qu'en remontant le fil des événements dans toute leur nuance et ambiguïté. Rien des processus menant au génocide n'était simple; ils ne le sont que lorsque nous décidons de les interpréter ainsi. Rien n'était déterminé et déterministe en 1990. Bien qu'il puisse nous répugner et même nous terroriser d'imaginer qu'un génocide ne naît pas d'autorités nécessairement infâmes ou de contextes nécessairement exceptionnels, c'est bien ce qu'il importe de comprendre. Les processus menant au génocide au Rwanda sont le fait d'autorités divisées, et dont les divisions, craintes, et aspirations mènent certaines à opter pour le génocide. Ils sont aussi le fait de cadres et pratiques ordinaires, de différentes formes de business as usual, qui peuvent finir par créer l'espace pour des événements terriblement hors de l'ordinaire. C'est du moins ce que de nombreux chercheurs dédiés à l'étude du Rwanda ont fini par conclure. Ce sont certainement les réflexions que nombre d'entre eux auraient apporté à l'entreprise de la commission.

Marie-Eve Desrosiers

École supérieure d'affaires publiques et internationales, Université d'Ottawa (Canada)

\footnotetext{
55 Rapport Duclert, p. 6.
} 


\section{Bibliographie}

ANDERSON Malcolm (1970), Government in France: An Introduction to Executive Power, Oxford/Toronto/New York, Pergamon Press.

CAPOCCIA Giovanni (2015), "Critical Junctures and Institutional Change », in J. MAHONEY et K. TheleN (dir.), Advances in Comparative-Historical Analysis, Cambridge, Cambridge University Press, pp. 147-179.

CHRÉTIEN Jean-Pierre (1995), « Rwanda : la propagande du génocide », in REPORTERS SANS FRONTIÈRES (dir.), Les Médias de la haine, Paris, La Découverte, pp. 22-56.

COMMISSION DE RECHERCHE SUR LES ARCHIVES FRANÇAISES RELATIVES AU RWANDA ET AU GÉNOCIDE DES TUTSI (2021), La France, le Rwanda et le génocide des Tutsi (1990-1994), Paris, Armand Colin.

DesRosiers Marie-Eve (2014), «Rethinking Political Rhetoric and Authority During Rwanda's First and Second Republics », Africa, 84(2), pp. 199-225.

(2020), «"Making Do" with Soft Authoritarianism in Pre-Genocide Rwanda », Comparative Politics, 52(4), pp. 557-579.

(à paraître), Elusive Control: Pre-Genocide Rwanda's Authoritarian Trajectory, manuscrit en cours d'évaluation.

Desrosiers Marie-Eve et Russell Aidan (2020), « Histories of Authority in the African Great Lakes: Trajectories and Transactions », Africa, 90(5), pp. 952-971.

DesRosiers Marie-Eve et Swedlund Haley J. (2019), «Rwanda's Post-Genocide Foreign Aid Relations: Revisiting Notions of Exceptionalism », African Affairs, 118(472), pp. 435-462.

FuJII Lee Ann (2009), Killing Neighbors. Webs of Violence in Rwanda, Ithaca/Londres, Cornell University Press.

GuichaOUA André (2010), Rwanda, de la guerre au génocide. Les politiques criminelles au Rwanda (1990-1994), Paris, La Découverte.

Hagmann Tobias et ReyntJens Filip (dir.) (2016), Aid and Authoritarianism in Africa: Development without Democracy, Londres, Zed Books.

KAJEGUHAKWA Valens (2001), Rwanda : de la terre de la paix à la terre de sang. Et après ?, Paris, Éditions Remi Perrin.

KESSLER Marie-Christine (1999), La Politique étrangère de la France. Acteurs et processus, Paris, Presses de Science Po.

KUPERMAN Alan J. (2004), «Provoking Genocide: A Revised History of the Rwandan Patriotic Front », Journal of Genocide Research, 6(1), pp. 61-84.

Lemarchand René (2009), The Dynamics of Violence in Central Africa, Philadelphia, University of Pennsylvania Press.

McDoom Omar Shahabudin (2021), "Radicalization as Cause and Consequence of Violence in Genocides and Mass Killings », Violence: An International Journal, 1(1), pp. 123-143. 
(2020), The Path to Genocide in Rwanda. Security, Opportunity, and Authority in an Ethnocratic State, Cambridge, Cambridge University Press.

MIRONKO Charles (2004), « Igitero: Means and Motive in the Rwandan Genocide », Journal of Genocide Research, 6(1), pp. 47-60.

MunYARUgerero François-Xavier (2003), Réseaux, pouvoirs, oppositions : la compétition politique au Rwanda, Paris, L'Harmattan.

Newbury Catharine et Newbury David (2000), «Bringing the Peasants Back In: Agrarian Themes in the Construction and Corrosion of Statist Historiography in Rwanda ", American Historical Review, 105(3), pp. 832-877.

PRUNIER Gérard (1993), «Éléments pour une histoire du Front patriote rwandais », Politique africaine, 51, pp. 121-138.

ReID Richard (2011), « Past and Presentism: the 'Precolonial' and the Foreshortening of African History », The Journal of African History, 52(2), pp. 135-155.

RIOT Thomas, BANCEL Nicolas et RUtAYISIRE Paul (2017), « Un art guerrier aux frontières des Grands Lacs. Aux racines dansées du Front Patriotique Rwandais », Politique africaine, 147, pp. 109-134.

UVIN Peter (1997), «Prejudice, Crisis, and Genocide in Rwanda », African Studies Review, 40(2), pp. 91 115.

(1998), Aiding Violence: The Development Enterprise in Rwanda, West Hartford, Kumarian Press.

VIDAL Claudine ([1985] 2005), "Situations ethniques au Rwanda », in J.-L. AMSELLE et É. M'BoKolo (dir.), Au coeur de l'ethnie. Ethnies, tribalisme et État en Afrique, Paris, La Découverte, pp. 167-184. 